\title{
Superconductivity in an Inhomogeneous Bundle of Metallic and Semiconducting Nanotubes
}

\author{
Ilya Grigorenko ${ }^{1}$ and Anvar Zakhidov ${ }^{2}$ \\ ${ }^{1}$ Physics Department, New York City College of Technology, The City University of New York, Brooklyn, NY 11201, USA \\ ${ }^{2}$ UTD-NanoTech Institute, The University of Texas at Dallas, Richardson, TX 75083-0688, USA \\ Correspondence should be addressed to Ilya Grigorenko; grigoren@gmail.com
}

Received 2 July 2013; Revised 6 September 2013; Accepted 6 September 2013

Academic Editor: Bobby G. Sumpter

Copyright (c) 2013 I. Grigorenko and A. Zakhidov. This is an open access article distributed under the Creative Commons Attribution License, which permits unrestricted use, distribution, and reproduction in any medium, provided the original work is properly cited.

\begin{abstract}
Using Bogoliubov-de Gennes formalism for inhomogeneous systems, we have studied superconducting properties of a bundle of packed carbon nanotubes, making a triangular lattice in the bundle's transverse cross-section. The bundle consists of a mixture of metallic and doped semiconducting nanotubes, which have different critical transition temperatures. We investigate how a spatially averaged superconducting order parameter and the critical transition temperature depend on the fraction of the doped semiconducting carbon nanotubes in the bundle. Our simulations suggest that the superconductivity in the bundle will be suppressed when the fraction of the doped semiconducting carbon nanotubes will be less than 0.5 , which is the percolation threshold for a two-dimensional triangular lattice.
\end{abstract}

Single wall carbon nanotubes (SWCNTs) represent a unique class of quasione-dimensional nanoscale systems exhibiting various interesting phenomena. Among other exciting features, it was demonstrated that individual single wall carbon nanotubes may have intrinsic superconducting properties [1]. However, because of their extremely small diameter (just few nanometers), and thus strongly one-dimensional character, the superconducting order parameter may have significant "phase slips" due to thermal and quantum fluctuations, leading to a finite conductivity in the system below the critical temperature $[2,3]$. Carbon nanotubes can also form bundles and ropes [4], with tens and hundreds of individual SWCNTs in the bundle, coupled to each other by dispersive Van der Waals forces. Such kind of system may exhibit reduced "phase slips" effects and as a result, much stronger conductivity drop below the critical temperature. The overall length of a SWCNT in the bundle also plays a significant role. For example, reducing the bundle's length to $300 \mathrm{~nm}$ may destroy the superconductivity in the system due to increasingly high quantum fluctuations [2]. Generally speaking, for nanoscale systems with the quantum level spacing approaching the superconducting gap energy $\Delta$, the superconductivity vanishes [5].
It is expected that doping of SWCNTs in a bundle by, for example, boron, may significantly improve their superconducting properties [6]. At a proper level of doping, the Fermi level may be at a one-dimensional singularity of the energy spectrum that gives a higher density of states (DOSs), that will lead to a higher critical temperature $T_{c}$. In particular, we assume here that such kind of mechanism of doping enhanced $T_{c}$ may be much better pronounced in the case of semiconducting SWCNTs, which may have higher DOS due to lower energy of the Van Hove singularities. This is in contrast to metallic SWCNTs, where singularities in the DOS are at higher energies and can be filled at much higher doping levels only (according to the Kataura plot [7]). This means that a bundle consisting of doped semiconducting nanotubes could be a much better superconductor compared to a bundle made of metallic SWCNTs.

However, synthesis of SWCNTs by currently known methods usually results in a mixture of semiconducting and metallic nanotubes. Since the nanotubes after the synthesis initially are not doped (or unintentionally slightly p-type doped, e.g., by oxygen of atmosphere), those are only metallic tubes, which may have superconducting transition, while semiconducting tubes will be "diluting" superconductivity in 
the bundle by the inverse proximity effect [8]. Upon doping (i.e., by electrochemical methods), the semiconducting tubes can become superconducting with a higher superconducting gap and thus a higher $T_{c}$ than in metallic nanotubes.

One may try to estimate a spatially averaged order parameter and the corresponding effective critical temperature for a bundle consisting of a mixture of these two types of SWCNTs. From an experimentalist's point of view, it is even more important to solve a bit more complex problem: for a given fraction of doped semiconducting SWCNTs in the bundle and the experimentally determined critical temperature $T_{c}$, to predict the critical temperature for a bundle, consisting only of doped semiconducting SWCNTs. It will be also interesting to know whether it is possible to obtain the critical temperature $T_{c}$ higher than in other carbon based nanostructures, like in alkali metal doped fullerenes.

Spatial variations of the superconducting order parameter are significant for nanoscale systems, including nanotubes $[9,10]$. In this work, we use a microscopic theory based on inhomogeneous Bogoliubov-de Gennes equations to establish how the superconducting properties of a bundle depend on the fraction of doped semiconducting nanotubes. We assume that the nanotubes in the bundle are approximately of the same radii and tightly packed making a triangular lattice in the bundle's transverse cross-section, with the primitive vectors $\vec{a}_{1}=R \vec{x}, \vec{a}_{2}=R \vec{x} / 2+\sqrt{3} / 2 R \vec{y}$. Here $\vec{x}, \vec{y}$ are the unit basis vectors, and $R$ is the average intertube distance. The lattice can be enumerated by indexes $(p, k)$, which correspond to the position of a nanotube $R_{p, k}=\vec{a}_{1} p+\vec{a}_{2} k$, but in this work we prefer to enumerate nanotubes in a $N \times N$ bundle using a single index through the mapping $i=p+$ $k N$, where $N$ is the number of nanotubes in the raw. The doped semiconducting nanotubes are assumed to occupy the fraction $a$ of the sites in the bundle, and the metallic nanotubes occupy the $1-a$ fraction of the sites. A full threedimensional description of a bundle taking into account the band structure and chirality of individual nanotubes would make simulations of the Bogoliubov-de Gennes equations too complex. On the other hand, assumption about the translation invariance along the longitude direction of the nanotubes would make the simulations marginally simpler, because one still needs to deal with quantum problem in three dimensions, but at the same time, this assumption would throw away the band structure difference between semiconducting and metallic nanotubes. In this study, we adopted a simplified two-dimensional picture which allows us to repeat our simulations several dozens of times for different arrangements of the nanotubes in the bundle. In the two-dimensional picture, the conduction electrons can stay in a nanotube or can hop to the neighboring nanotubes (sites). In principle, there may be three different hoping constants, with the hopping matrix elements $t^{i i^{\prime}}$ equal to either $t^{\mathrm{mm}}, t^{\mathrm{ms}}$, and $t^{\mathrm{ss}}$, corresponding to the hopping between metallic-metallic ( $\mathrm{mm})$, metallic-semiconducting ( $\mathrm{ms})$, or semiconducting-semiconducting (ss) nanotubes. Moreover, these parameters may significantly fluctuate from one site to another, due to variations of the intertube distance $R$ or the mismatch between SWCNTs of different radii and chirality, but in this work, we neglect all these fluctuations. In the superconducting regime, Cooper pairs can be formed within nanotubes and can also hop from one tube to another.

For the description of the system, we utilized the Hamiltonian form:

$$
\begin{aligned}
H_{0}= & \sum_{\left\langle\mathbf{r}_{i}, \mathbf{r}_{j}\right\rangle, \sigma} t^{i, j} c_{\mathbf{r}_{i}, \sigma}^{\dagger} c_{\mathbf{r}_{j}, \sigma}-\mu \sum_{\left\langle\mathbf{r}_{i}\right\rangle, \sigma} c_{\mathbf{r}_{i}, \sigma}^{\dagger} c_{\mathbf{r}_{i}, \sigma} \\
& +\sum_{\left\langle\mathbf{r}_{i}\right\rangle} U_{\text {int }}^{i}\left(\mathbf{r}_{i}\right) n_{\downarrow}\left(\mathbf{r}_{i}\right) n_{\uparrow}\left(\mathbf{r}_{i}\right) \\
& +\sum_{\left\langle\mathbf{r}_{i}, \mathbf{r}_{j}\right\rangle, \sigma, \sigma^{\prime}} V_{\text {int }}^{i j} n_{\sigma}\left(\mathbf{r}_{i}\right) n_{\sigma^{\prime}}\left(\mathbf{r}_{j}\right)
\end{aligned}
$$

where the quantum-mechanical operator $c_{\mathbf{r}_{i}, \sigma}^{\dagger}$ creates an electron on site $i$ (using the single indexing), the operator $c_{\mathbf{r}_{j}, \sigma}$ eliminates an electron from the site $j$, and $n_{\sigma}\left(\mathbf{r}_{i}\right)=$ $c_{\mathbf{r}_{i}, \sigma}^{\dagger} c_{\mathbf{r}_{i}, \sigma}$ represents the electron density on site $i$ with the spin polarization $\sigma$. The electron spin, $\sigma$, can point up or down. $U_{\text {int }}^{i}$ is the onsite interaction potential. This term in a case of attractive interaction $U_{\text {int }}^{i}<0$ may lead to pairing in the nanotube $i . V_{\text {int }}^{i j}$ is a strength of the coupling between electrons localized at neighboring tubes $i$ and $j$.

Using the Bogoliubov transformation, which diagonalizes the Hamiltonian equation (1), we arrive to inhomogeneous Bogoliubov-de Gennes equations for the quasiparticle amplitudes on the lattice $i$ sites $\left(u_{n}\left(\mathbf{r}_{i}\right), v_{n}\left(\mathbf{r}_{i}\right)\right)$ [11]:

$$
\left(\begin{array}{cc}
\widehat{\xi} & \widehat{\Delta} \\
\widehat{\Delta}^{*} & -\widehat{\xi}^{*}
\end{array}\right)\left(\begin{array}{l}
u_{n}\left(\mathbf{r}_{i}\right) \\
v_{n}\left(\mathbf{r}_{i}\right)
\end{array}\right)=E_{n}\left(\begin{array}{l}
u_{n}\left(\mathbf{r}_{i}\right) \\
v_{n}\left(\mathbf{r}_{i}\right)
\end{array}\right),
$$

where the kinetic operator $\widehat{\xi}$ and the superconducting order parameter $\widehat{\Delta}$ can be represented as

$$
\begin{aligned}
& \widehat{\xi} u_{n}\left(\mathbf{r}_{i}\right)=-\sum_{\boldsymbol{\delta}} t^{i, j} u_{n}\left(\mathbf{r}_{i}+\boldsymbol{\delta}\right)+\left(V^{s}\left(\mathbf{r}_{i}\right)-\mu\right) u_{n}\left(\mathbf{r}_{i}\right) \\
& \widehat{\Delta} v_{n}\left(\mathbf{r}_{i}\right)=\sum_{\boldsymbol{\delta}} \Delta_{\boldsymbol{\delta}}\left(\mathbf{r}_{i}\right) v_{n}\left(\mathbf{r}_{i}+\boldsymbol{\delta}\right)+\Delta_{\boldsymbol{s}}\left(\mathbf{r}_{i}\right) v_{n}\left(\mathbf{r}_{i}\right)
\end{aligned}
$$

where $\delta$ are the nearest neighbor vectors for a triangular lattice, $V^{s}\left(\mathbf{r}_{i}\right)$ is the mean field (Hartree) potential, $\mu$ is the chemical potential. $\widehat{\Delta}_{\mathbf{s}}$ is the conventional, $s$-type order parameter. One should solve (2) together with the selfconsistency conditions as follows:

$$
\begin{aligned}
\Delta_{\boldsymbol{\delta}}\left(\mathbf{r}_{i}\right)=\sum_{n} \frac{V_{\text {int }}^{i j}}{2} & \left(u_{n}\left(\mathbf{r}_{i}+\boldsymbol{\delta}\right) v_{n}^{*}\left(\mathbf{r}_{i}\right)\right. \\
& \left.+u_{n}\left(\mathbf{r}_{i}\right) v_{n}^{*}\left(\mathbf{r}_{i}+\boldsymbol{\delta}\right)\right) \tanh \left(\frac{E_{n}}{2 k_{B} T}\right),
\end{aligned}
$$


where the pairing strength $V_{\text {int }}^{i j}$ may depend on the type of CN at the sites $i$ and $j$. The $s$-type order parameter (within a given nanotube $i$ ) is simply

$$
\begin{aligned}
\Delta_{\mathbf{s}}\left(\mathbf{r}_{i}\right)= & \sum_{n} \frac{U_{\text {int }}^{i}}{2}\left(u_{n}\left(\mathbf{r}_{i}\right) v_{n}^{*}\left(\mathbf{r}_{i}\right)\right. \\
& \left.+u_{n}\left(\mathbf{r}_{i}\right) v_{n}^{*}\left(\mathbf{r}_{i}\right)\right) \tanh \left(\frac{E_{n}}{2 k_{B} T}\right) .
\end{aligned}
$$

Note that the summation in (4), (5) is done over the positive eigenvalues $E_{n}$ only. The amplitudes $u_{n}\left(\mathbf{r}_{i}\right), v_{n}\left(\mathbf{r}_{i}\right)$ obey the constraints $\int d \mathbf{r}\left(\left|u_{n}\left(\mathbf{r}_{i}\right)\right|^{2}+\left|v_{n}\left(\mathbf{r}_{i}\right)\right|^{2}\right)=1$ for any $n$ (normalization) and $\sum_{n}\left(\left|u_{n}\left(\mathbf{r}_{i}\right)\right|^{2}+\left|v_{n}\left(\mathbf{r}_{i}\right)\right|^{2}\right)=1$ for any $i, i$ being the site index of the triangular lattice.

Here, we assumed the same constant hopping parameter $t$ between any type of nanotubes. In this work, we assumed that the pairing may only happen between electrons in the same nanotube, therefore neglecting a much weaker pairing mechanism between neighboring nanotubes. In principle, a weak attraction mechanism may stimulate the formation of a Cooper pair with one electron in one nanotube and the second electron in one of its nearest neighbors. This may result in coexisting order parameters in the system. The coexistence of order parameters with different symmetries, was studied, for example, for uranium-based superconducting materials [12]. The possibility of such pairing on a triangular lattice may result in unconventional superconducting properties. For example, a 2D triangular lattice was recently considered as a test-bed for a possibility of $f$-wave spin-triplet superconductivity [13].

We studied how the spatially averaged superconducting order parameter $\langle\Delta\rangle=\left(1 / N^{2}\right) \sum_{k} \Delta_{s}\left(\mathbf{r}_{k}\right)$ depends on the fraction of doped semiconducting SWCNTs $a$ in a $N \times$ $N$ bundle at different temperatures. For this purpose, we generated $P=50$ realizations for a given number of randomly placed semiconducting nanotubes in the bundle. Other nanotubes in the bundle are assumed to be metallic. To model different superconducting pairing strengths for different types of nanotubes, we set $U_{\text {int }}^{i}=2 t$ for the doped semiconducting nanotubes and $U_{\text {int }}^{i}=0.68 t$ for the metallic ones, so the doped semiconducting nanotubes have a higher critical temperature. For simplicity, we set $\mu=0$ (half-filled band). In our simulations we considered $16 \times 16$ nanotubes in the bundle, forming a triangular lattice.

The results of simulations are shown in Figure 1. At $T=0$, the spatially averaged order parameter scales approximately as a square root of the fraction of doped semiconducting SWCNTs in the bundle $\langle\Delta\rangle \propto\left(\Delta^{s}-\Delta^{m}\right) \sqrt{a}+\Delta^{m}$. Here $\Delta^{m}$ and $\Delta^{s}$ are the order parameters for pure metallic and doped semiconducting bundles. Note the convexity of the dependence. However, at finite temperatures, which are between the critical temperature of a pure metallic $T_{c}^{m}$ and pure doped semiconducting $T_{c}^{s}$ bundles, the averaged order parameter vanishes much faster with the decreasing of $a$. For example, at $T=0.32 t$, which is close to the critical temperature of a pure semiconducting SWCNT bundle $T_{c}^{s} \approx$ $0.35 t$, the order parameter decreases very quickly with the decreasing of $a$ and almost vanishes at $a \approx 0.5$ (see Figure 1).

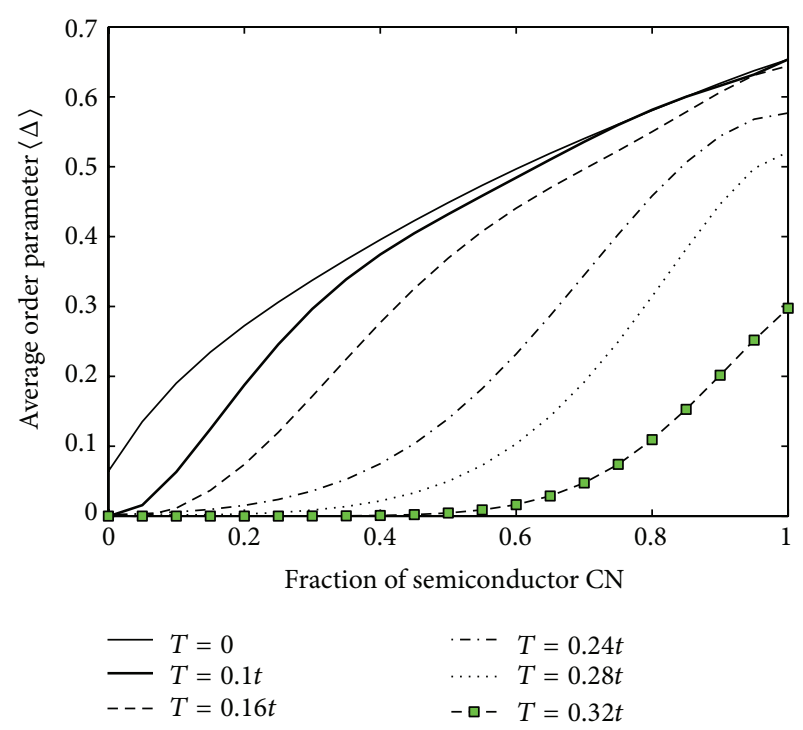

FIGURE 1: Spatially averaged superconducting order parameter $\langle\Delta\rangle$ (in units of $t$ ) as a function of the fraction of doped semiconducting nanotubes $a$ at different temperatures.

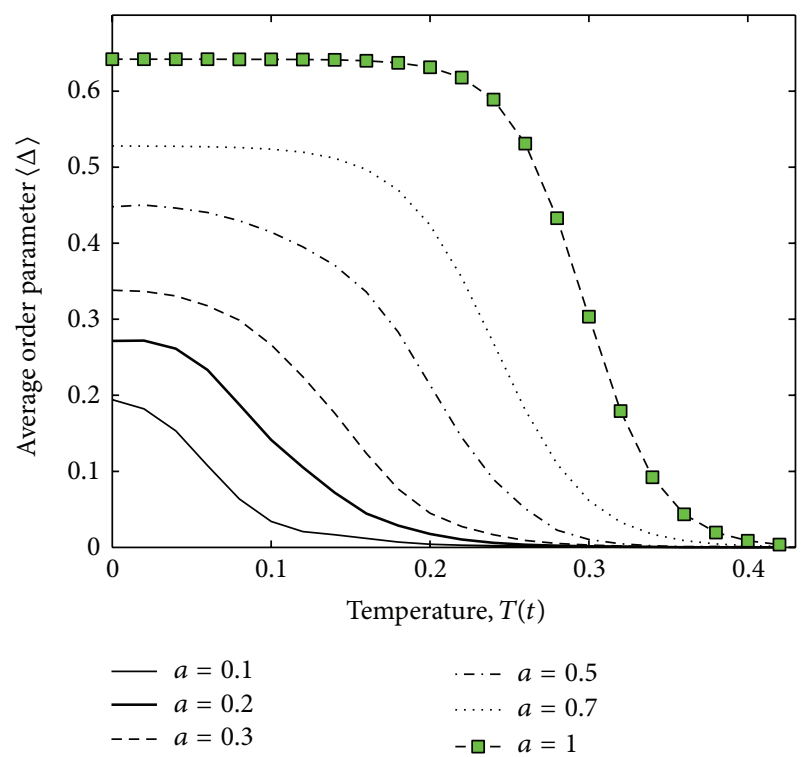

FIGURE 2: Spatially averaged superconducting order parameter $\langle\Delta\rangle$ (in units of $t$ ) as a function of temperature for different values of $a$ (the fraction of doped semiconducting nanotubes).

In Figure 2, we plot $\langle\Delta\rangle$ as a function of temperature for several values of $a$. One can clearly see how the order parameter vanishes above the critical temperature. Note that with the lowering of the fraction of semiconducting nanotubes $a$, the temperature dependence of the order parameter shows less pronounced phase transition because of the "dirty" nature of inhomogeneous spatial distribution of the pairing properties that is similar to dirty superconducting transition in case of large concentration of impurities and in alloys. We also would like to emphasize that the systems 
under consideration are finite; therefore, the order parameter vanishes not abruptly as in the BCS theory for an infinite system, even for the homogeneous case $a=1$. Because of the presence of the temperature tails in Figure 2, we define the critical temperature as a temperature at which the order parameter drops to just $5 \%$ of its value at $T=0$.

Using the data plotted in Figure 2, we calculated how the critical temperature $T_{c}$ depends on the concentration of doped semiconducting SWCNTs. We used $t=4.8 \times$ $10^{-3} \mathrm{eV}$ to be consistent with the data in $[1,6]$, so $a=$ 0.6 will correspond to $T_{c} \approx 15 \mathrm{~K}$. In Figure 3 , we plot the averaged critical temperature $T_{c}$ as a function of the fraction of semiconducting CNs. First, one can clearly see a nonlinear dependence of the $T_{c}$. Second, in Figure 3 one can observe that for $a<0.5$, the critical temperature changes with the change of $a$ with a steeper slope than for $a>0.5$. For $a<0.5$, the weaker superconducting metallic CNs are arranged in bigger islands, which surround and isolate doped semiconducting nanotubes from each other. The inverse proximity effect of low $T_{c}$ metallic tubes will significantly reduce the averaged order parameter in the system. Note, $a=0.5$ corresponds to the site percolation threshold on the plane triangular lattice [14]. If one would go beyond the mean field description and would take into account the phase fluctuations of the order parameter, the dependence would be even steeper, because of the enhanced phase fluctuations in relatively well-isolated doped semiconducting nanotubes, surrounded by metallic nanotubes. For $a<0$, this will result in a rapid decrease of the spatially averaged order parameter.

According to our model for an optimally doped bundle consisting of $100 \%$ semiconducting SWCNTs, the $T_{c}$ should increase to the unsuppressed $T_{c}$ of $19-20 \mathrm{~K}$ (see Figure 3 ). The effect of $T_{c}$ suppression similar to the one discussed here has been observed in alkali doped fullerene molecular alloys of $A_{x}\left(C_{60}\right)_{x}\left(C_{70}\right)_{1-x}$ [15], and adding non-superconducting component, that is, $C_{70}$ molecules, which do not show any superconducting pairing due to symmetry reasons and probably due to weaker electron-phonon coupling, strongly suppressed $T_{c}$ from $19 \mathrm{~K}$ in $100 \% C_{60}$, that is, in $K_{3} C_{60}$ to $T_{c}=10 \mathrm{~K}$ in $20 \%$ substituted $C_{70}$ alloy. The experiments with selectively separated metallic and semiconducting SWCNTs which now become available by new methods of effective separation will allow to check the validity simple model of presented here and to clarify the role of quantum fluctuations, which has not been accounted here.

One has to note that even for relatively small bundles $16 \times 16$, the physical properties have relatively small variations for different realizations of the spatial distributions of semiconducting nanotubes. To support this observation, we plot a whole set of the spatially averaged order parameters for 50 random configurations of the nanotubes for different temperatures. We choose the equal number of the metallic and semiconducting nanotubes in the bundle $a=0.5$. In Figure 4, one can see that there are relatively limited variations of the order parameter corresponding to different configurations of the nanotubes. The overall dispersion is limited to approximately $0.05 t$ at zero temperature and becomes relatively significant at temperatures approaching

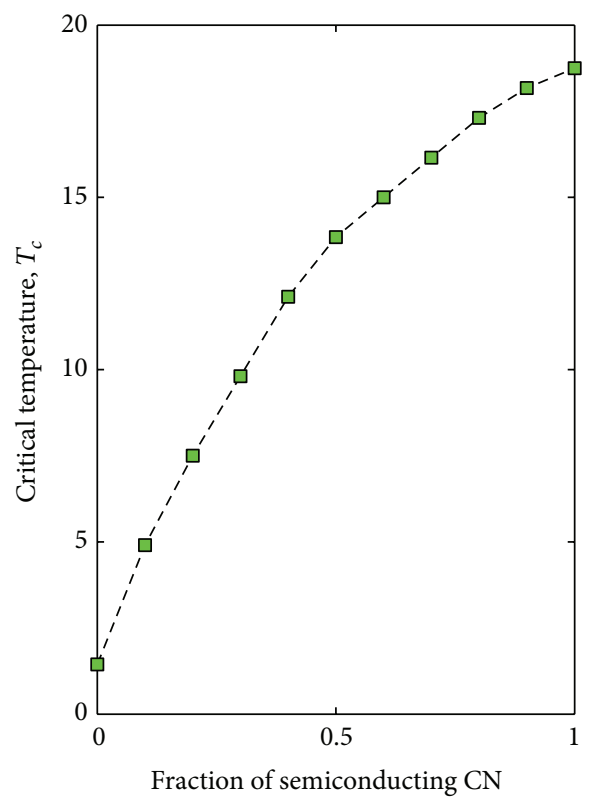

FIgURE 3: Critical temperature $T_{c}$ as a function of the fraction of doped semiconducting nanotubes $a$. Note a steeper slope for $a<$ 0.5 ( $a=0.5$ is the site percolation limit in two dimensions on a triangular lattice).

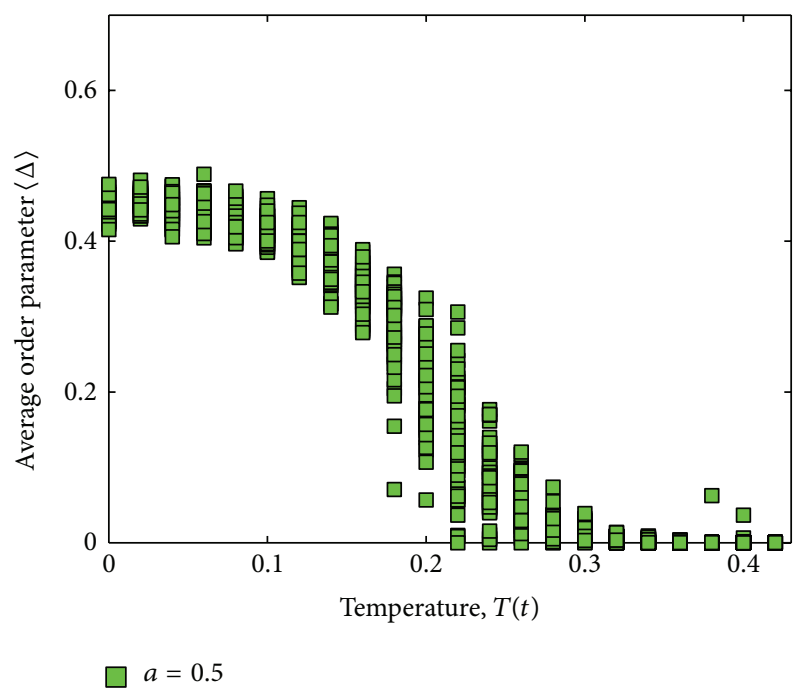

FIGURE 4: A set of spatially averaged order parameters (in units of $t$ ) as a function of temperature used for statistical average over different configurations at the percolation regime $(a=0.5)$. Note that the distribution has a limited variation width.

the critical temperature. For experimentalists, this means that one needs to take extra care about the measurements of small samples near the critical temperature.

In Figure 5, we plot a particular realization of the spatial distribution of the superconducting order parameter (in units of $t)$ at the percolation regime $(a=0.5)$ and zero temperature. In Figure 5, red dots mark the triangular lattice of a $16 \times$ 


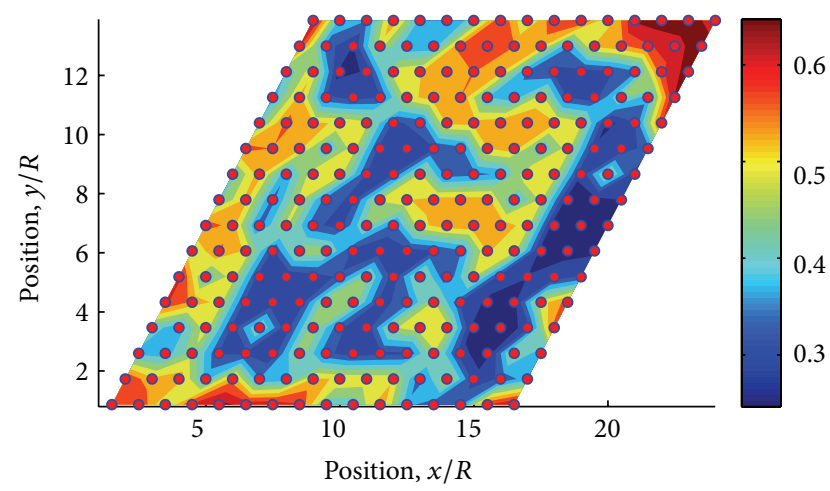

FIGURE 5: A particular realization of the spatial distribution of the superconducting order parameter (in units of $t$ ) at the percolation regime $(a=0.5)$ and zero temperature. Red dots mark the triangular lattice of a $16 \times 16$ bundle.

16 bundle. One can see the formation of big clusters of nanotubes with the same superconducting properties. For the measurements of the transverse conductivity of such bundles at finite temperatures, one should expect to see a dramatic drop of the conductivity for $a>0.5$, that is, above the percolation threshold for the triangular lattice. At this concentration, the semiconducting nanotubes will likely create a connected network within a finite bundle of nanotubes. We propose that this prediction will be tested experimentally.

In conclusion, we introduced a mean field microscopic model to describe superconductivity in a bundle of a mixture of carbon nanotubes of different superconducting properties. We have studied the dependence of the spatially averaged superconducting gap $\langle\Delta\rangle$ on the fraction of doped semiconducting SWCNT (with a higher pairing strength) in the bundle at different temperatures. Note that for inhomogeneous nanoscale systems, the dependence $T_{c}\left(\langle\Delta\rangle_{T=0}\right)$ for different concentrations $a$ may be nonlinear, as a manifestation of the breakdown of the bulk BCS theory. Indeed, our calculations of $T_{c}\left(\langle\Delta\rangle_{T=0}\right)$ show that for $a>0.5$ the dependence $T_{c}=$ $T_{c}\left(\langle\Delta\rangle_{T=0}\right)$ has less steep slope. The reason is that the bundle is a highly inhomogeneous system with a pronounced inverse proximity effect. At $a<0.5$, below the site percolation threshold for a 2D triangular lattice, the bundle can be seen as a collection of finite islands of "good" superconductors (doped semiconducting nanotubes), diluted by normal or weakly superconducting material (metallic nanotubes). Such islands demonstrate significantly the suppressed superconductivity, even in the mean field description, due to the enhanced inverse proximity effect.

Note that our mean field BdG model is unable to predict and properly describe quantum phase fluctuations of the order parameter in isolated doped semiconducting nanotubes, where the superconductivity will be suppressed even stronger. Future research using, for example, GinzburgLandau inhomogeneous equations $[2,16-18]$ is necessary to describe such kind of effects. Because the dynamics of Cooper pairs in doped carbon nanotubes can be more close to the diffusive regime, the Usadel equations can be applied to calculate the finite conductivity at $T \ll T_{c}[19]$.

\section{References}

[1] Z. K. Tang, L. Zhang, N. Wang et al., "Superconductivity in 4 angstrom single-walled carbon nanotubes," Science, vol. 292, no. 5526, pp. 2462-2465, 2001.

[2] G. V. Pai, E. Shimshoni, and N. Andrei, "Resistivity of inhomogeneous superconducting wires," Physical Review B, vol. 77, no. 10, Article ID 104528, 2008.

[3] D. S. Golubev and A. D. Zaikin, "Thermally activated phase slips in superconducting nanowires," Physical Review B, vol. 78, no. 14, Article ID 144502, 2008.

[4] M. Kociak, A. Y. Kasumov, S. Guéron et al., "Superconductivity in ropes of single-walled carbon nanotubes," Physical Review Letters, vol. 86, no. 11, pp. 2416-2419, 2001.

[5] D. C. Ralph, C. T. Black, and M. Tinkham, "Spectroscopic measurements of discrete electronic states in single metal particles," Physical Review Letters, vol. 74, no. 16, pp. 3241-3244, 1995.

[6] N. Murata, J. Haruyama, J. Reppert et al., "Superconductivity in thin films of boron-doped carbon nanotubes," Physical Review Letters, vol. 101, no. 2, Article ID 027002, 2008.

[7] H. Kataura, Y. Kumazawa, Y. Maniwa et al., "Optical properties of single-wall carbon nanotubes," Synthetic Metals, vol. 103, no. 1-3, pp. 2555-2558, 1999.

[8] S. Guéron, H. Pothier, N. O. Birge, D. Esteve, and M. H. Devoret, "Superconducting proximity effect probed on a mesoscopic length scale," Physical Review Letters, vol. 77, no. 14, pp. 30253028, 1996.

[9] J.E. Han and V.H. Crespi, "Discrete transverse superconducting modes in nanocylinders," Physical Review B, vol. 69, no. 21, Article ID 214526, pp. 1-214526, 2004.

[10] A. A. Shanenko, M. D. Croitoru, and F. M. Peeters, "Nanoscale superconductivity: nanowires and nanofilms," Physica $C$, vol. 468, no. 7-10, pp. 593-598, 2008.

[11] M. Ma and P. A. Lee, "Localized superconductors," Physical Review B, vol. 32, no. 9, pp. 5658-5667, 1985.

[12] P. Kumar and P. Wole, "Two-component order-parameter model for pure and thorium-doped superconducting $\mathrm{UBe}_{13}$ ", Physical Review Letters, vol. 59, no. 17, pp. 1954-1957, 1987.

[13] H. Ikeda, Y. N. Isikawa, and K. Yamadai, "Possibility of f-wave spin-triplet superconductivity in the $\mathrm{CoO}_{2}$ superconductor: a case study on a $2 \mathrm{D}$ triangular lattice in the repulsive Hubbard model," Journal of the Physical Society of Japan, vol. 73, pp. 1720, 2004.

[14] M. F. Sykes and J. W. Essam, "Exact critical percolation probabilities for site and bond problems in two dimensions," Journal of Mathematical Physics, vol. 5, no. 8, pp. 1117-1127, 1964.

[15] K. Yoshino, X. H. Yin, S. Morita, and A. A. Zakhidov, "Difference in doping effects of C60 and C70 in poly(3hexylthiophene)," Japanese Journal of Applied Physics, vol. 32, no. 1, pp. L140-L143, 1993.

[16] A. Zharov, A. Lopatin, A. E. Koshelev, and V. M. Vinokur, "Microscopic theory of thermal phase slips in clean narrow superconducting wires," Physical Review Letters, vol. 98, no. 19, Article ID 197005, 2007.

[17] D. S. Golubev and A. D. Zaikin, "Quantum tunneling of the order parameter in superconducting nanowires," Physical Review B, vol. 64, Article ID 014504, 2001. 
[18] A. Bezryadin, "Quantum suppression of superconductivity in nanowires," Journal of Physics, vol. 20, no. 4, Article ID 043202, 2008.

[19] A. V. Semenov, P. A. Krutitskii, and I. A. Devyatov, "Microscopic theory of phase slip in a narrow durty superconducting strip," JETP Letters, vol. 92, no. 11, pp. 762-766, 2010. 

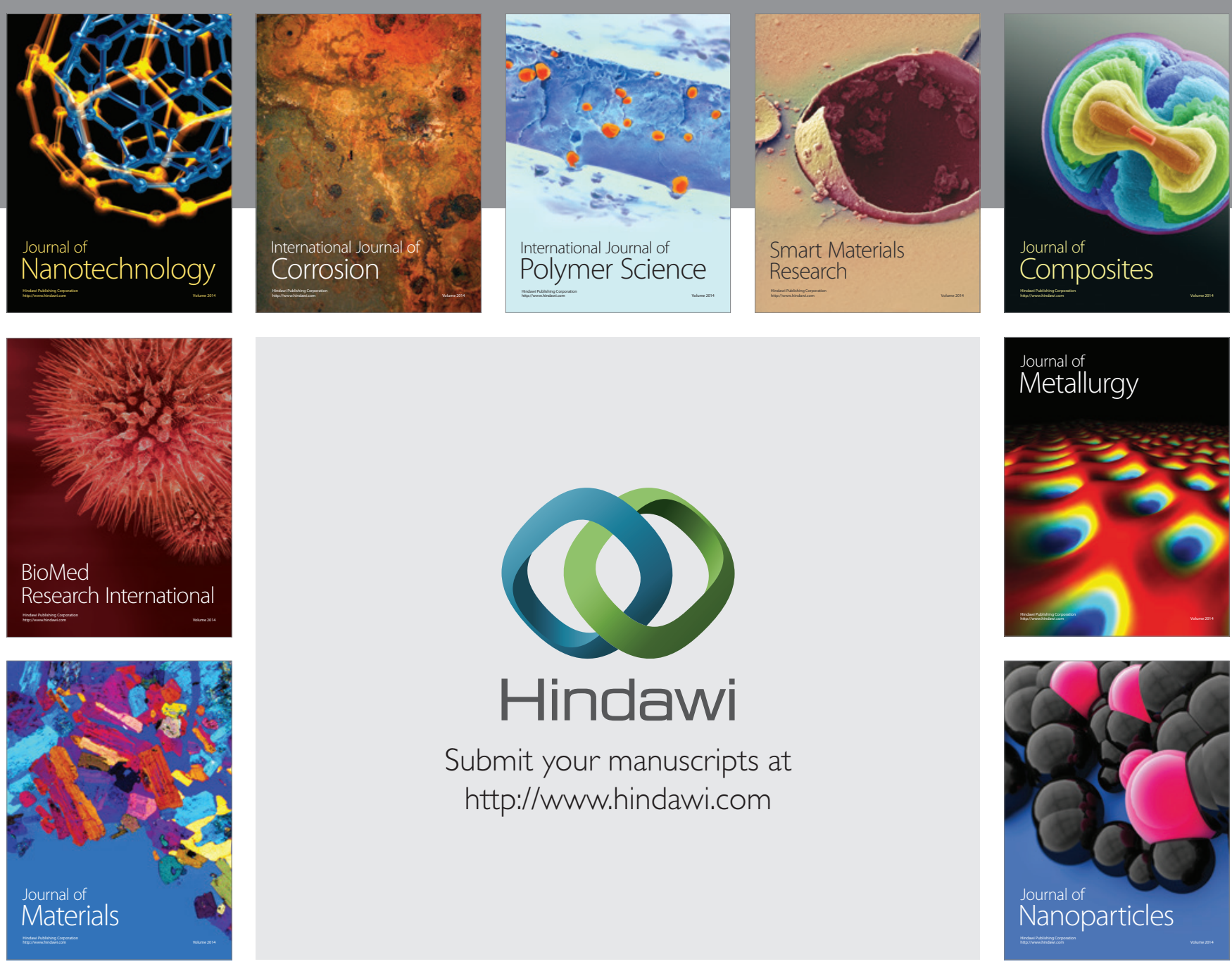

Submit your manuscripts at http://www.hindawi.com
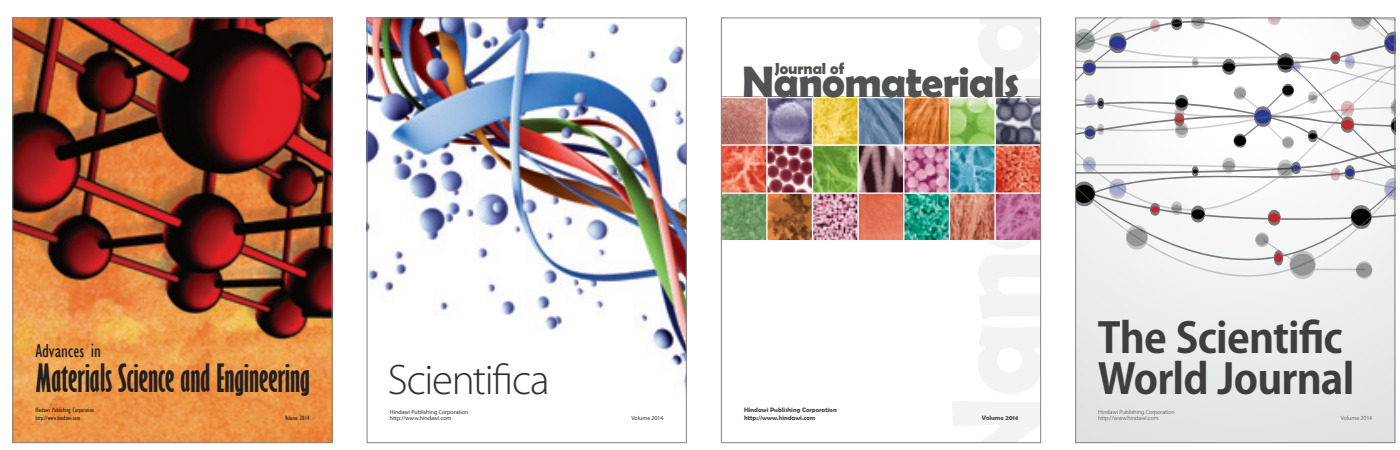

\section{The Scientific World Journal}
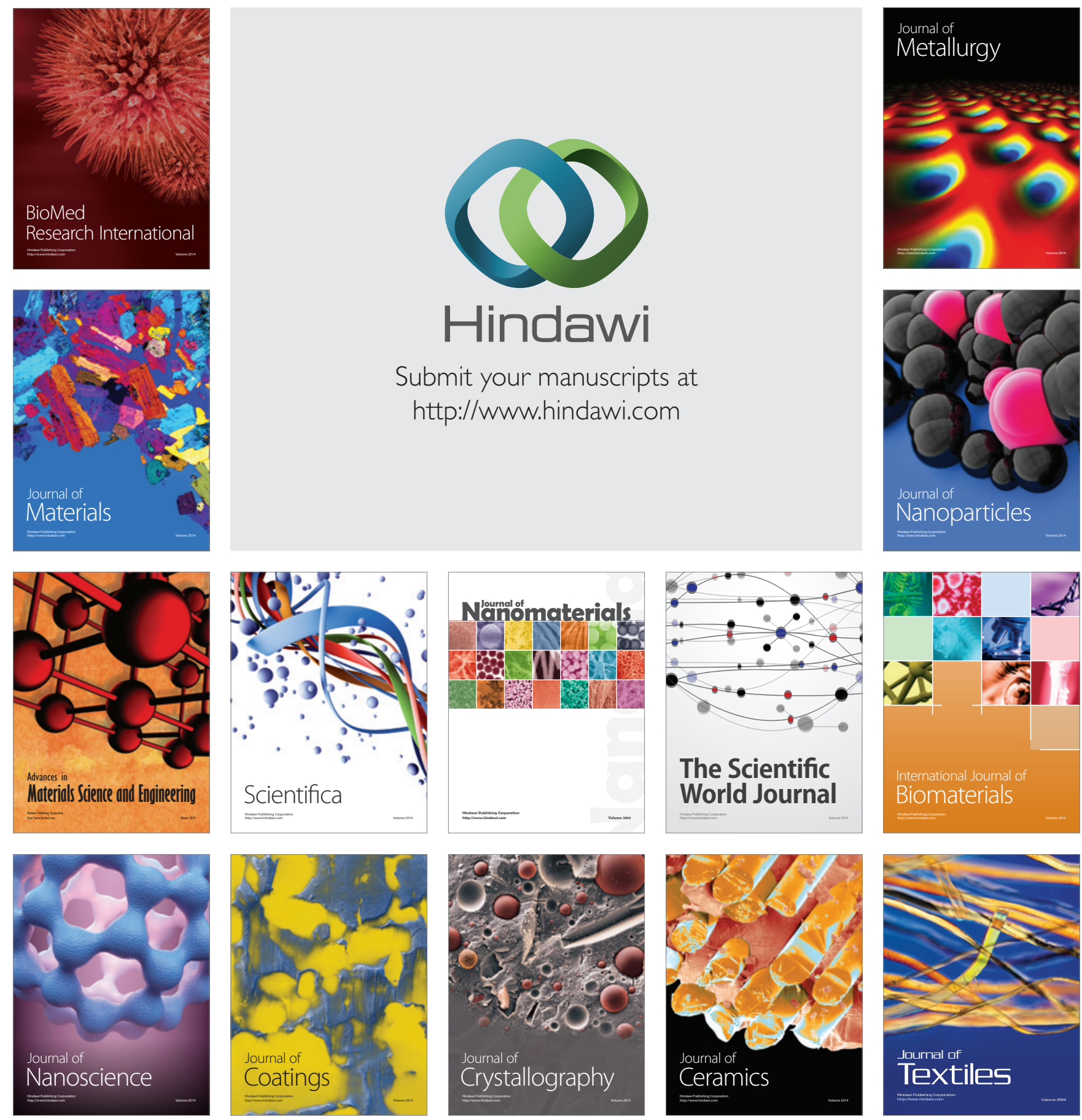